\title{
Access to Photon Fragmentation Functions in Hadronic Jet Production
}

\author{
Tom Kaufmann $^{a}$, Asmita Mukherjee ${ }^{b}$, Werner Vogelsang ${ }^{a}$ \\ ${ }^{a}$ Institute for Theoretical Physics, Tübingen University, Auf der Morgenstelle 14, \\ 72076 Tübingen, Germany \\ ${ }^{b}$ Department of Physics, Indian Institute of Technology Bombay, \\ Powai, Mumbai 400076, India
}

\begin{abstract}
We argue that the process $p p \rightarrow($ jet $\gamma) X$, for which a photon is observed inside a fully reconstructed jet and is treated as part of the jet, offers new probes of the so far little known fragmentation functions for photons. We present a next-to-leading-order QCD calculation of the cross section for this process in the limit that the jet is relatively narrow. We also investigate the background resulting from the two-photon decay of neutral pions. We present numerical results relevant for possible measurements at the LHC and at RHIC. These suggest that $p p \rightarrow($ jet $\gamma$ ) $X$ should provide clean access to the photon fragmentation functions, provided an efficient suppression of the background is available in experiment.
\end{abstract}




\section{Introduction}

The production of photons with high transverse momentum $p_{T}$ in hadronic collisions is of fundamental importance in today's particle and nuclear physics. Foremost, it may serve as a tool for determining the gluon distributions of the scattering hadrons, thanks to the presence and dominance of the leading order (LO) Compton subprocess $q g \rightarrow \gamma q$. Photons also provide sensitive probes of the medium produced in collisions of heavy ions, being able to traverse and escape the medium with little attenuation. Finally, photon signals play an important role in studies of physics within and beyond the Standard Model, with the process $p p \rightarrow \gamma \gamma X$ through production and decay of a Higgs boson and the recent indication of a $750 \mathrm{GeV}$ diphoton excess seen by the ATLAS and CMS collaborations [1] at the LHC arguably being among the most well-known examples.

When used in searches of new physics phenomena, photon "signal" processes are invariably affected by backgrounds from more mundane Standard Model sources. In view of this it is clear that a good quantitative theoretical understanding of photon production in high-energy hadronic collisions is crucial. For photons produced in a process characterized by a large momentum transfer, perturbative-QCD methods may be used. As was discussed a long time ago [2], in perturbative high- $p_{T}$ processes there are two production mechanisms for photons. The photon may be directly produced in the hard scattering process through its "pointlike" QED coupling to a quark. Such contributions are usually referred to as "direct". On the other hand, photons may also emerge in jet fragmentation, when a quark, antiquark, or gluon emerging from a QCD hardscattering process fragments into a photon plus a number of hadrons. The need for introducing such a "photon fragmentation" contribution is physically motivated by the fact that the photon may result, for example, from conversion of a high- $p_{T} \rho$ meson. Furthermore, at higher orders, the perturbative direct component contains divergencies from configurations where the photon and a final-state quark become collinear. These are long-distance contributions that naturally signify the need for non-perturbative fragmentation functions into which they can be absorbed.

Using the single-inclusive process $p p \rightarrow \gamma X$ as an example, QCD perturbation theory thus predicts the following schematic structure of the factorized cross section:

$$
d \sigma=\sum_{a, b} f_{a} \otimes f_{b} \otimes\left[d \hat{\sigma}_{a b}^{\gamma}+\sum_{c} d \hat{\sigma}_{a b}^{c} \otimes D_{c}^{\gamma}\right] .
$$

Here the sums run over all partons (quarks, antiquarks and gluons). The part involving the sum over partons $c$ is the fragmentation component, while the other part is the direct one. Each part has its own partonic hard-scattering cross sections for producing either directly a photon, or a parton $c$ that subsequently fragments into the photon as described by the fragmentation functions $D_{c}^{\gamma} \equiv D_{c}^{\gamma}(z, \mu)$, where $z$ is the fraction of the parton's momentum picked up by the photon and $\mu$ the scale at which the fragmentation function is probed. Both the direct and the fragmentation parts involve parton distribution functions in the proton, denoted by $f_{a}$ and $f_{b}$. The symbols $\otimes$ represent appropriate integrations over momentum fractions of the various participating partons.

Theoretical calculations of photon production cross sections in hadronic collisions thus rely on three ingredients: 
(i) knowledge of parton distributions,

(ii) precision calculations of the partonic cross sections, and

(iii) parton-to-photon fragmentation functions.

The parton distribution functions of protons are by now very well known. A lot of work has gone into (ii) over the past three decades. For single-inclusive photon production, full next-toleading order (NLO) computations of the partonic cross sections for the direct [3] as well as for the fragmentation part [4] have been performed, and even all-order resummations of large logarithmic threshold corrections are available for both parts [5].

Rather little is known, on the other hand, about the photon fragmentation functions. Some information is available from measurements at LEP [6], but with rather large uncertainties. Theoretical predictions [7-10] for the photon fragmentation functions are compatible with these sparse data. Based on such sets of $D_{c}^{\gamma}$, one estimates that fragmentation photons contribute about 10$30 \%$ to the cross section for $p p \rightarrow \gamma X$ in the fixed-target regime [11]. At high-energy colliders, the fragmentation contribution is typically predicted to be only $10 \%$ or less [12]. The reason for this is that at colliders one introduces so-called isolation cuts. To isolate a photon candidate, one basically demands that the hadronic energy in a cone around the photon be limited to a certain value. Isolation suppresses the large background from $\pi^{0} \rightarrow \gamma \gamma$ decay and also reduces the fragmentation contribution [12] since it basically confines it to very high values of the fragmentation variable $z$.

At any rate, the size of the fragmentation contribution arguably presents the largest uncertainty in predictions of photon production cross sections. In the present paper, we propose a new promising method to experimentally access the $D_{c}^{\gamma}$ in hadronic collisions. The idea is to identify a photon as part of a fully reconstructed jet. One measures the transverse momenta of the photon and the jet, $p_{T}$ and $p_{T}^{\text {jet }}$, respectively. As we shall show, their ratio,

$$
z_{\gamma} \equiv \frac{p_{T}}{p_{T}^{\text {jet }}}
$$

offers direct scans of the fragmentation functions $D_{c}^{\gamma}(z, \mu)$ in $z$. This is an advantage over the inclusive cross section $p p \rightarrow \gamma X$, which may also provide information on the photon fragmentation functions [13] but typically samples them over a broad region in $z$. We develop the formalism for the "same-side" photon-jet observable to NLO accuracy. Using the approximation of relatively narrow reconstructed jets, we are able to derive analytical results for the relevant NLO cross sections. In our previous publication [14] (see also [15-17] for related work), we have already presented the corresponding NLO calculation for the process $p p \rightarrow$ (jet $h) X$, where $h$ denotes an identified hadron inside the jet, and demonstrated that this process opens a promising window on the determination of the parton-to-hadron fragmentation functions $D_{c}^{h}$. Evidently, the background from $\pi^{0} \rightarrow \gamma \gamma$ decay mentioned above will also be relevant in our case and hence will also be analyzed in our paper.

We stress that the observable we have in mind is different from the "away-side" photon-jet correlations considered in Ref. [18] and provides a kinematically simpler and more direct access to the $D_{c}^{\gamma}$. In particular, in our case it is natural to divide the cross section for $p p \rightarrow($ jet $\gamma) X$ by the 
single-inclusive jet one for $p p \rightarrow$ jet $X$, in which case many theoretical uncertainties related to the choice of parton distributions or (initial-state) factorization scale will cancel out. We also note that same-side photon-jet observables have been studied in the contexts of $e^{+} e^{-}$annihilation $[10,19]$ and $e p$ collisions [20], where they were shown to provide access to the $D_{q}^{\gamma}$. However, the accuracy achievable in experiment is rather limited for these reactions, as the LEP results [6] show. More importantly, a drawback of these processes is that the gluon-to-photon fragmentation function can be probed only indirectly by evolution or higher-order corrections. This is different for the process $p p \rightarrow($ jet $\gamma) X$ which probes all photon fragmentation functions in the same hadronic environment as relevant for photon signals at colliders.

Our paper is organized as follows. In Sec. 2 we present our NLO calculation of $p p \rightarrow($ jet $\gamma) X$. We shall see that the results can be formulated in terms of simple photonic "jet" functions. Section 3 describes calculations of the pion decay background, taking into account basic experimental considerations concerning pion reconstruction. In Sec. 4 we present phenomenological results for $p p \rightarrow($ jet $\gamma) X$ relevant for collisions at RHIC and the LHC.

\section{Same-side jet-plus-photon production at NLO}

We consider a high- $p_{T}$ photon produced inside a jet. Our approach will be to consider the photon as part of the jet. To this end, we follow Ref. $[10,19]$ to cluster hadrons and photons "democratically" into mixed hadronic and electromagnetic jets, a procedure that may be applied to any of the commonly used jet algorithms [21-23]. In terms of a perturbative-QCD calculation it means that the photon is included as an additional parton.

At lowest order in QCD, for $z_{\gamma}<1$, there are only fragmentation contributions to the cross section for $p p \rightarrow($ jet $\gamma) X$. It is this feature that makes the cross section a powerful probe of the photon fragmentation functions. Our strategy for performing the NLO calculation is based on our previous work [14] on the process $p p \rightarrow$ (jet $h$ ) $X$. A key element of that study was the "narrow jet approximation (NJA)" [24] (see also [25]), which assumes that the parameter $\mathcal{R}$ specifying the jet "opening" is relatively small. This allows for an analytical calculation of the NLO partonic cross sections. The NJA is accurate up to corrections of order $\mathcal{R}^{2}$. As was shown in Refs. [24], this is sufficient for essentially all jet opening parameters used in experiment.

In the language of Ref. [14], the NLO factorized cross section for $p p \rightarrow($ jet $\gamma) X$ may be written as

$$
\begin{aligned}
\frac{d \sigma^{p p \rightarrow(\mathrm{jet} \gamma) X}}{d p_{T}^{\mathrm{jet}} d \eta^{\mathrm{jet}} d z_{\gamma}} & =\sum_{\substack{a, b, c \in \\
\{q, \bar{q}, g, \gamma\}}} \int_{x_{a}^{\min }}^{1} d x_{a} f_{a}\left(x_{a}, \mu_{F}\right) \int_{x_{b}^{\min }}^{1} d x_{b} f_{b}\left(x_{b}, \mu_{F}\right) \int_{z_{c}^{\min }}^{1} d z_{c} \frac{d \hat{\sigma}_{a b}^{c}\left(\hat{s}, \hat{p}_{T}, \hat{\eta}, \mu_{F}, \mu_{F}^{\prime}, \mu_{R}\right)}{d p_{T}^{\mathrm{jet}} d \eta^{\mathrm{jet}}} \\
& \times \sum_{\substack{d \in \in \\
\{q, \bar{q}, g, \gamma\}}} j_{c \rightarrow d}\left(z_{c}, \frac{\mathcal{R} p_{T}^{\mathrm{jet}}}{\mu_{F}^{\prime}}\right) \sum_{\substack{c^{\prime} \in \\
\{, \bar{q}, g, \gamma\}}} \int_{z_{\gamma}}^{1} \frac{d z_{p}}{z_{p}} \tilde{j}_{d \rightarrow c^{\prime}}\left(z_{p}, \frac{\mathcal{R} p_{T}^{\mathrm{jet}}}{\mu_{F}^{\prime \prime}}\right) \mathcal{D}_{c^{\prime}}^{\gamma}\left(\frac{z_{\gamma}}{z_{p}}, \mu_{F}^{\prime \prime}\right)
\end{aligned}
$$

Apart from the variable $z_{\gamma}$ defined in (2) the cross section is specified by the jet's transverse 
momentum $p_{T}^{\text {jet }}$ and rapidity $\eta^{\text {jet }}$, the latter being equal to the photon rapidity $\eta$ in the NJA. In Eq. (3) we have furthermore defined

$$
\begin{aligned}
& x_{a}^{\min }=\frac{p_{T}^{\text {jet }} \mathrm{e}^{\eta^{\mathrm{jet}}}}{\sqrt{S}-p_{T}^{\mathrm{jet}} \mathrm{e}^{-\eta^{\mathrm{jet}}}}, \quad x_{b}^{\mathrm{min}}=\frac{x_{a} p_{T}^{\mathrm{jet}} \mathrm{e}^{-\eta^{\mathrm{jet}}}}{x_{a} \sqrt{S}-p_{T}^{\mathrm{jet}} \mathrm{e}^{\eta^{\mathrm{jet}}}}, \\
& z_{c}^{\mathrm{min}}=\frac{p_{T}^{\mathrm{jet}}}{\sqrt{S}}\left(\frac{\mathrm{e}^{\eta^{\mathrm{jet}}}}{x_{a}}+\frac{\mathrm{e}^{-\eta^{\mathrm{jet}}}}{x_{b}}\right),
\end{aligned}
$$

where $\sqrt{S}$ is the hadronic c.m.s. energy. The various partonic variables are $\hat{s}=x_{a} x_{b} S, \hat{p}_{T}=$ $p_{T}^{\text {jet }} / z_{c}, \hat{\eta}=\eta^{\text {jet }}-\frac{1}{2} \log \left(x_{a} / x_{b}\right)$.

Equation (3) has been written in such a way that it includes both the direct and the fragmentation contributions. This is achieved by introducing the function

$$
\mathcal{D}_{c^{\prime}}^{\gamma}(z, \mu) \equiv \delta(1-z) \delta_{c^{\prime} \gamma}+D_{c^{\prime}}^{\gamma}(z, \mu)\left(1-\delta_{c^{\prime} \gamma}\right),
$$

where the first part corresponds to the direct contribution and the second part to the fragmentation one. The partonic cross sections $d \hat{\sigma}_{a b}^{c}$ (with $c=\gamma, q, \bar{q}, g$ ) in (3) are the usual NLO single-inclusive photon/parton cross sections as computed in Refs. [3] and [4], respectively. Their perturbative expansions to NLO, but to lowest order in the electromagnetic coupling $\alpha$, read

$$
\begin{aligned}
d \hat{\sigma}_{a b}^{\gamma} & =\alpha \alpha_{s}\left[d \hat{\sigma}_{a b}^{\gamma,(0)}+\frac{\alpha_{s}}{\pi} d \hat{\sigma}_{a b}^{\gamma,(1)}+\mathcal{O}\left(\alpha_{s}^{2}\right)\right], \\
d \hat{\sigma}_{a b}^{c \neq \gamma} & =\alpha_{s}^{2}\left[d \hat{\sigma}_{a b}^{c,(0)}+\frac{\alpha_{s}}{\pi} d \hat{\sigma}_{a b}^{c,(1)}+\mathcal{O}\left(\alpha_{s}^{2}\right)\right] .
\end{aligned}
$$

Evidently, in order to compute the cross section for production of photons inside a jet, one needs corresponding partonic cross sections for this observable. These necessarily differ from the singleinclusive cross sections $d \hat{\sigma}_{a b}^{\gamma}, d \hat{\sigma}_{a b}^{c}$. As was shown in Ref. [14], it is relatively straightforward to convert the $d \hat{\sigma}_{a b}^{\gamma}, d \hat{\sigma}_{a b}^{c}$ into the cross sections that we need for same-side photon (or hadron) plus jet production. This is achieved by introducing suitable perturbative jet functions $j_{c \rightarrow d}$ and $\tilde{j}_{d \rightarrow c^{\prime}}$. The former describe the formation of a jet "consisting" of parton $d$ which has taken the fraction $z_{c}$ of the parent parton's momentum, while the latter represent the "partonic fragmentation" of parton $d$ to parton $c^{\prime}$ inside the jet, with momentum fraction $z_{p}$.

Before discussing the jet functions in more detail, we note that we have introduced several scales in Eq. (3): The renormalization scale $\mu_{R}$, the initial-state factorization scale $\mu_{F}$ and two final-state factorization scales $\mu_{F}^{\prime}$ and $\mu_{F}^{\prime \prime}$, respectively. Of the latter two, $\mu_{F}^{\prime}$ is an artifact of the way we organize our calculation, formulating the cross section in terms of single-inclusive parton cross sections. $\mu_{F}^{\prime}$ was originally introduced in the mass factorization procedure for the $d \hat{\sigma}_{a b}^{c}$. Now, however, it cancels exactly between the $d \hat{\sigma}_{a b}^{c}$ and the jet functions $j_{c \rightarrow d}$ due to the fact that we foremost define our observable by requiring an infrared- and collinear safe jet in the final state. Hence, $\mu_{F}^{\prime}$ is not present in the final result. The actual final-state factorization scale which survives in our final answer is $\mu_{F}^{\prime \prime}$. This scale appears because we are asking for an observed photon in the final state as part of the jet. For details, see Ref. [14].

Throughout this paper (and as anticipated in Eq. (6)), we will always work to lowest order $\mathcal{O}(\alpha)$ in the electromagnetic coupling $\alpha$. This means that the initial partons $a, b$ in Eq. (3) will 
never be photons in our calculations since the requirement that there also is a photon in the final state would make the corresponding contribution to the cross section $\mathcal{O}\left(\alpha^{2}\right)$. This holds true even for the photon fragmentation part since the fragmentation functions $D_{c}^{\gamma}$ always provide an additional power of $\alpha$. In order to specify the jet functions, it is therefore sufficient to just consider a fixed initial partonic state $a b(a, b \neq \gamma)$ in Eq. (3) and disregard the parton distribution functions. Schematically, we then have the expression

$$
\sum_{c, d, c^{\prime}} d \hat{\sigma}_{a b}^{c} \otimes j_{c \rightarrow d} \otimes \tilde{j}_{d \rightarrow c^{\prime}} \otimes \mathcal{D}_{c^{\prime}}^{\gamma} .
$$

The labels $c, d, c^{\prime}$ run over all QCD partons, but can also represent a photon. The jet functions with both $c \neq \gamma$ and $c^{\prime} \neq \gamma$ contribute only to the fragmentation part. They are identical to those appearing in our earlier calculation [14] for hadron production inside jets, where they were given to NLO. To LO, they are all diagonal, $j_{q \rightarrow q}=j_{g \rightarrow g}=\delta\left(1-z_{c}\right)$ and $\tilde{j}_{q \rightarrow q}=\tilde{j}_{g \rightarrow g}=\delta\left(1-z_{p}\right)$. This shows that at this order the cross section for $p p \rightarrow($ jet $\gamma) X$ directly probes the photon fragmentation functions at value $z=z_{\gamma}$.

Starting at NLO, there is also a direct component of the cross section for photon-in-jet production. The corresponding contributions are always associated with either $c=\gamma$ or $c^{\prime}=\gamma$. Being direct contributions, they always come with the piece $\propto \delta(1-z)$ in $\mathcal{D}_{c^{\prime}}^{\gamma}$ in $(5)$. We now discuss the various possibilities at $\mathcal{O}(\alpha)$ :

(i) Case $c=c^{\prime}=\gamma$ : Here the expression in (7) becomes

$$
\sum_{d} \underbrace{d \hat{\sigma}_{a b}^{\gamma}}_{\mathcal{O}\left(\alpha \alpha_{s}\right)} \otimes j_{\gamma \rightarrow d} \otimes \tilde{j}_{d \rightarrow \gamma} \otimes \underbrace{\mathcal{D}_{\gamma}^{\gamma}}_{\mathcal{O}(1)}
$$

As indicated, the partonic cross sections $d \hat{\sigma}_{a b}^{\gamma}$ start at order $\mathcal{O}\left(\alpha \alpha_{s}\right)$ (see Eq. (6)). Since all jet functions for $d \neq \gamma$ are of order $\mathcal{O}(\alpha)$, they would give rise to higher-order contributions in $\alpha$. Hence, only $d=\gamma$ and $j_{\gamma \rightarrow \gamma}=\delta\left(1-z_{c}\right)$ and $\tilde{j}_{\gamma \rightarrow \gamma}=\delta\left(1-z_{p}\right)$ is possible at $\mathcal{O}(\alpha)$. Thus, this part gives precisely the direct part of the full NLO single-inclusive photon production cross section. However, because of the $\delta$-functions from $\tilde{j}_{\gamma \rightarrow \gamma}$ and $\mathcal{D}_{\gamma}^{\gamma}$, the entire piece comes with a factor $\delta\left(1-z_{\gamma}\right)$, as is easily seen by insertion into Eq. (3). This in turn implies that for any measurement of the cross section carried out at $z_{\gamma}<1$ this part of the cross section will not contribute. ${ }^{\ddagger}$

In cases (ii) and (iii) we have $c=q$ and $c^{\prime}=\gamma$ so that the expression in (7) becomes

$$
\sum_{d} \underbrace{d \hat{\sigma}_{a b}^{q}}_{\mathcal{O}\left(\alpha_{s}^{2}\right)} \otimes j_{q \rightarrow d} \otimes \tilde{j}_{d \rightarrow \gamma} \otimes \underbrace{\mathcal{D}_{\gamma}^{\gamma}}_{\mathcal{O}(1)} .
$$

To lowest order in $\alpha$, we can then either have $d=\gamma$ or $d=q$ in Eq. (3). Thus we further distinguish:

\footnotetext{
${ }^{\ddagger}$ We note that the region $z_{\gamma} \rightarrow 1$ could be interesting when isolation cuts are imposed on the photon. In this region we have a photon carrying most of the jet's energy and accompanied by very little hadronic energy. For such studies at very high $z_{\gamma}$ it may be preferred to integrate over a range of $z_{\gamma}$ that includes the endpoint at $z_{\gamma}=1$, in which case the contributions $\propto \delta\left(1-z_{\gamma}\right)$ will need to be kept.
} 
(ii) Case $c=q, c^{\prime}=\gamma, d=\gamma$ : We have $\tilde{j}_{\gamma \rightarrow \gamma}=\delta\left(1-z_{p}\right)$ and, in the $\overline{\mathrm{MS}}$ scheme,

$$
j_{q \rightarrow \gamma}(z, \lambda)=-\frac{\alpha}{2 \pi} e_{q}^{2}\left[P_{\gamma q}(z) \log \left(\lambda^{2}(1-z)^{2}\right)+z\right]
$$

where $e_{q}$ is the quark's fractional charge, and where

$$
P_{\gamma q}(z)=\frac{1+(1-z)^{2}}{z} .
$$

This contribution is again proportional to $\delta\left(1-z_{\gamma}\right)$ and does not contribute to measurements at $z_{\gamma}<1$. However, this becomes different in

(iii) case $c=q, c^{\prime}=\gamma, d=q$, where $j_{q \rightarrow q}=\delta\left(1-z_{c}\right)$ and, in the $\overline{\mathrm{MS}}$ scheme and for the anti- $k_{t}$ jet algorithm:

$$
\tilde{j}_{q \rightarrow \gamma}^{\overline{\mathrm{MS}}}(z, \lambda)=\frac{\alpha}{2 \pi} e_{q}^{2}\left[P_{\gamma q}(z) \log \left(\lambda^{2} z^{2}(1-z)^{2}\right)+z\right] .
$$

The contribution associated with this jet function is, at this order, the only direct part of the cross section that is present at $z_{\gamma}<1$. It is genuinely NLO, and its logarithmic dependence on the scale $\mu_{F}^{\prime \prime}$ correctly compensates the scale dependence associated with the inhomogeneous piece [7-10] in the evolution of the $D_{c}^{\gamma}$.

The final possibility is

(iv) case $c=g, c^{\prime}=\gamma$ : Here we need to 'convert' a gluon exiting the hard scattering to an observed photon. This may happen through the combination $j_{g \rightarrow q} \tilde{j}_{q \rightarrow \gamma}$. The product of these functions is $\mathcal{O}\left(\alpha \alpha_{s}\right)$, which, when combined with the $\alpha_{s}^{2}$ from the hard process is an order too high in $\alpha_{s}$, that is, beyond NLO. The only contribution for $c=g$ arises when the photon is produced in fragmentation, as discussed above.

We collect all jet functions in the Appendix, both for the fragmentation and for the direct cases, and for various types of jet algorithms. From the above discussion, we conclude that as long as we stay at $z_{\gamma}<1$ case (iii) generates the only non-vanishing direct contributions at $\mathcal{O}(\alpha)$ and NLO. We observe that the corresponding jet function in Eq. (12) shows a logarithmic divergence at $z \rightarrow 1$. A behavior of this type is quite familiar for NLO direct (pointlike) contributions in hadronic processes involving photons, where it is also exhibited by the corresponding photonic coefficient functions for space-like or time-like photon structure functions $[8,26]$. This can become an issue, in principle, due to the fact that the coefficient functions appear directly in the structure function, potentially creating an unphysical divergent behavior of the structure function at high Bjorken- $x$. However, as was shown in Refs. [8, 26], for photon structure functions the situation may be remedied by adopting a factorization scheme different from the $\overline{\mathrm{MS}}$ scheme for the pointlike

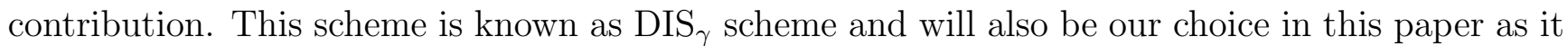
leads to an overall better perturbative stability. Using the results of [8] the scheme transformation is easily performed, and we find for the jet function in the $\mathrm{DIS}_{\gamma}$ scheme:

$$
\begin{aligned}
\tilde{j}_{q \rightarrow \gamma}^{\mathrm{DIS}}(z, \lambda) & \equiv \tilde{j}_{q \rightarrow \gamma}^{\overline{\mathrm{MS}}}(z, \lambda)-\frac{\alpha}{2 \pi} e_{q}^{2}\left[P_{\gamma q}(z) \log \left(z^{2}(1-z)\right)-2 \frac{1-z}{z}\right] \\
& =\frac{\alpha}{2 \pi} e_{q}^{2} P_{\gamma q}(z)\left[\log \left(\lambda^{2}(1-z)\right)+1\right] .
\end{aligned}
$$


All other jet functions remain unaffected by the transformation. Interestingly, we find that even in the $\operatorname{DIS}_{\gamma}$ scheme a logarithmic term $\propto \log (1-z)$ remains in the jet function. The reason for this can be traced back to the fact that, compared to the inclusive structure function $F_{1}^{\gamma}$, the phase space for our photon-in-jet observable is different, being less inclusive and hence giving rise to two factors of $\propto \log (1-z)$ in the $\overline{\mathrm{MS}}$ scheme, only one of which is subtracted when transforming to the $\mathrm{DIS}_{\gamma}$ scheme. In any case, the remaining logarithm in (13) may be eliminated by a scale choice $\left(\mu_{F}^{\prime \prime}\right)^{2} \propto\left(1-z_{\gamma}\right)$. As mentioned, the jet function in (13) refers to the anti- $k_{t}$ jet algorithm. The results for other algorithms may be found in the Appendix for the $\overline{\mathrm{MS}}$ scheme and may then be transformed to the $\mathrm{DIS}_{\gamma}$ scheme in the same fashion as above.

For later reference, we finally note that the cross section (3) may also be expressed in terms of photon kinematics,

$$
\frac{d \sigma^{p p \rightarrow(\mathrm{jet} \gamma) X}}{d p_{T} d \eta d z_{\gamma}}\left(p_{T}, \eta, z_{\gamma}\right)=\frac{1}{z_{\gamma}} \frac{d \sigma^{p p \rightarrow(\text { jet } \gamma) X}}{d p_{T}^{\text {jet }} d \eta^{\text {jet }} d z_{\gamma}}\left(p_{T}^{\text {jet }}=\frac{p_{T}}{z_{\gamma}}, \eta, z_{\gamma}\right) .
$$

\section{Pion decay background}

Production of $\pi^{0}$ with the subsequent decay $\pi^{0} \rightarrow \gamma \gamma$ (with a branching ratio of $\approx 98.8 \%$ [27]) provides the largest source of unwanted background photons. There are two main components of this background $[28,29]$. First, at high pion transverse momentum, the two decay photons can become almost collinear and may not be resolved as two separate photons but rather as a single electromagnetic signal. We refer to this part of the background as "B1". Secondly, even when the two decay photons are well separated in angle, the decay may be very asymmetric in energy. It may happen that one of the decay photons is relatively soft, so that it is below the threshold for detection. In this case, only the high-energy decay photon will be seen and misinterpreted as a single prompt photon. This part of the background, denoted as "B2", is relevant toward lower transverse momentum, where the pion production cross section is large.

Using our previous calculation [14], it is relatively straightforward to estimate the sizes of B1 and B2. From the axial anomaly prediction [30] in the chiral limit we have a constant decay matrix element, so that the normalized differential decay rate follows just from phase space considerations:

$$
\frac{1}{\Gamma} \frac{d \Gamma}{d E_{1}}=\frac{1}{\left|\vec{P}_{\pi}\right|},
$$

where $E_{1}$ is the energy of one of the decay photons and $\vec{P}_{\pi}$ denotes the pion three-momentum in the hadronic c.m.s. As we are interested in the angular separation of the two photons, we relate $E_{1}$ to $\cos \theta_{12}$, where $\theta_{12}$ is the angle between the momenta of the two decay photons:

$$
\cos \theta_{12}=1-\frac{m_{\pi}^{2}}{2 E_{1}\left(E_{\pi}-E_{1}\right)} .
$$

We then obtain

$$
\frac{1}{\Gamma} \frac{d \Gamma}{d \cos \theta_{12}}=\frac{m_{\pi}^{2}}{\left|\vec{P}_{\pi}\right|} \frac{1}{\left(1-\cos \theta_{12}\right)^{2} \sqrt{E_{\pi}^{2}-\frac{2 m_{\pi}^{2}}{1-\cos \theta_{12}}}},
$$


where $E_{\pi}$ is the pion energy. For B1 we only need to impose the condition that $\theta_{12}$ be smaller than the angle the detector can resolve, so that the photons will be seen as a single electromagnetic signal. This happens when

$$
1-\cos \theta_{12} \leq \frac{\delta \phi^{2}+\delta \eta^{2}}{2 \cosh ^{2} \eta} \equiv \frac{\rho^{2}}{2 \cosh ^{2} \eta}
$$

where $\delta \phi$ and $\delta \eta$ are the detector resolutions in azimuthal angle and rapidity, respectively. To estimate B1 numerically, we simply have to integrate the normalized decay spectrum (17) subject to the constraint (18), i.e.

$$
\int_{1-\rho^{2} /\left(2 \cosh ^{2} \eta\right)}^{1-2\left(1-\beta^{2}\right)} d \cos \theta_{12} \frac{1}{\Gamma} \frac{d \Gamma}{d \cos \theta_{12}}=\frac{1}{\left|\vec{P}_{\pi}\right|} \sqrt{E_{\pi}^{2}-4 m_{\pi}^{2} \frac{\cosh ^{2} \eta}{\rho^{2}}} \Theta\left(E_{\pi}^{2}-4 m_{\pi}^{2} \frac{\cosh ^{2} \eta}{\rho^{2}}\right)
$$

where $\Theta(x)$ denotes the Heaviside function, and multiply the result by the cross section for $p p \rightarrow$ $\left(\right.$ jet $\left.\pi^{0}\right) X$ from our previous calculation [14]. Here we have introduced the Lorentz factor $\beta=$ $\left|\vec{P}_{\pi}\right| / E_{\pi}$.

For B2, for which one of the two decay photons is not detected and the other photon has transverse momentum $p_{T}$, one needs to take into account that the decaying pion must have had a correspondingly higher transverse momentum or energy. This gives rise to a convoluted structure of the cross section for this part of the background. Denoting the energy threshold for photon detection by $\varepsilon$, we have

$$
\frac{d \sigma^{\mathrm{B} 2}}{d \eta^{\mathrm{jet}} d p_{T}^{\gamma} d z_{\gamma}}=\int_{p_{T}^{\gamma}}^{\infty} \frac{d p_{T}^{\pi}}{p_{T}^{\pi}} \frac{d \sigma^{\left(\mathrm{jet} \pi^{0}\right)}}{d \eta^{\mathrm{jet}} d p_{T}^{\pi} d z_{\pi}} \frac{1}{\Gamma} \frac{d \Gamma\left(x=p_{T}^{\gamma} / p_{T}^{\pi}\right)}{d x},
$$

where

$$
\frac{1}{\Gamma} \frac{d \Gamma(x)}{d x}=\frac{2}{\beta} \Theta\left[\frac{1+\beta}{2}-x\right] \Theta\left[x-\max \left(1-\frac{\varepsilon}{E_{\pi}}, \frac{1}{2}\left(1+\sqrt{1-\frac{4 m_{\pi}^{2} \cosh ^{2} \eta}{\rho^{2} E_{\pi}^{2}}}\right)\right)\right]
$$

We have introduced the variable $x$ as the energy fraction of one photon with respect to the pion energy, $E_{1}=x E_{\pi}$, and hence $E_{2}=(1-x) E_{\pi}$. Furthermore, $p_{T}^{\pi}$ is the transverse momentum of the decaying pion and $z_{\pi} \equiv p_{T}^{\pi} / \hat{p}_{T}^{\text {jet }}$ is defined in analogy to Eq. (2), with $\hat{p}_{T}^{\text {jet }}$ the transverse momentum of the jet containing the pion. Clearly, we have $E_{\pi}=E_{\gamma}+E_{2}$ and hence $p_{T}^{\gamma}=x p_{T}^{\pi}$, since we assume that the other photon's energy, $E_{2}$, is not seen by the detector as it falls below the detector's energy threshold. A further consequence of this is that the photon with energy $E_{2}$ is not counted toward the jet energy. As a result, we have $p_{T}^{\text {jet }}=x \hat{p}_{T}^{\text {jet }}$. Combining these relations, we find that $z_{\pi}=z_{\gamma}$.

We now rewrite the integration in Eq. (20) in terms of the energy fraction $x$ and use Eq. (14) to express everything in terms of the jet kinematics. Using $z_{\pi}=z_{\gamma}$ we finally arrive at

$$
\frac{d \sigma^{\mathrm{B} 2}}{d p_{T}^{\mathrm{jet}} d \eta^{\mathrm{jet}} d z_{\gamma}}=\int_{0}^{1} d x \frac{d \sigma^{\left(\mathrm{jet} \pi^{0}\right)}}{d \hat{p}_{T}^{\mathrm{jet}} d \eta^{\mathrm{jet}} d z_{\pi}} \frac{1}{\Gamma} \frac{d \Gamma(x)}{d x}
$$


The integration limits in this equation are implemented implicitly in the definition of $\frac{1}{\Gamma} \frac{d \Gamma(x)}{d x}$ via Heaviside functions, see Eq. (21). They follow from the conditions that the decay be kinematically possible, that the unobserved decay photon must have energy below $\varepsilon$, and that the two photons have an opening angle larger than the detector resolution. Explicitly, one has

$$
1+\frac{m_{\pi}^{2}}{4 E_{\gamma}^{2}} \leq x^{-1} \leq \min \left(1+\frac{\varepsilon}{E_{\gamma}}, 1+\frac{m_{\pi}^{2}}{\rho^{2} p_{T}^{2}}\right),
$$

with $E_{\gamma}=p_{T} \cosh \eta$ the observed photon's energy.

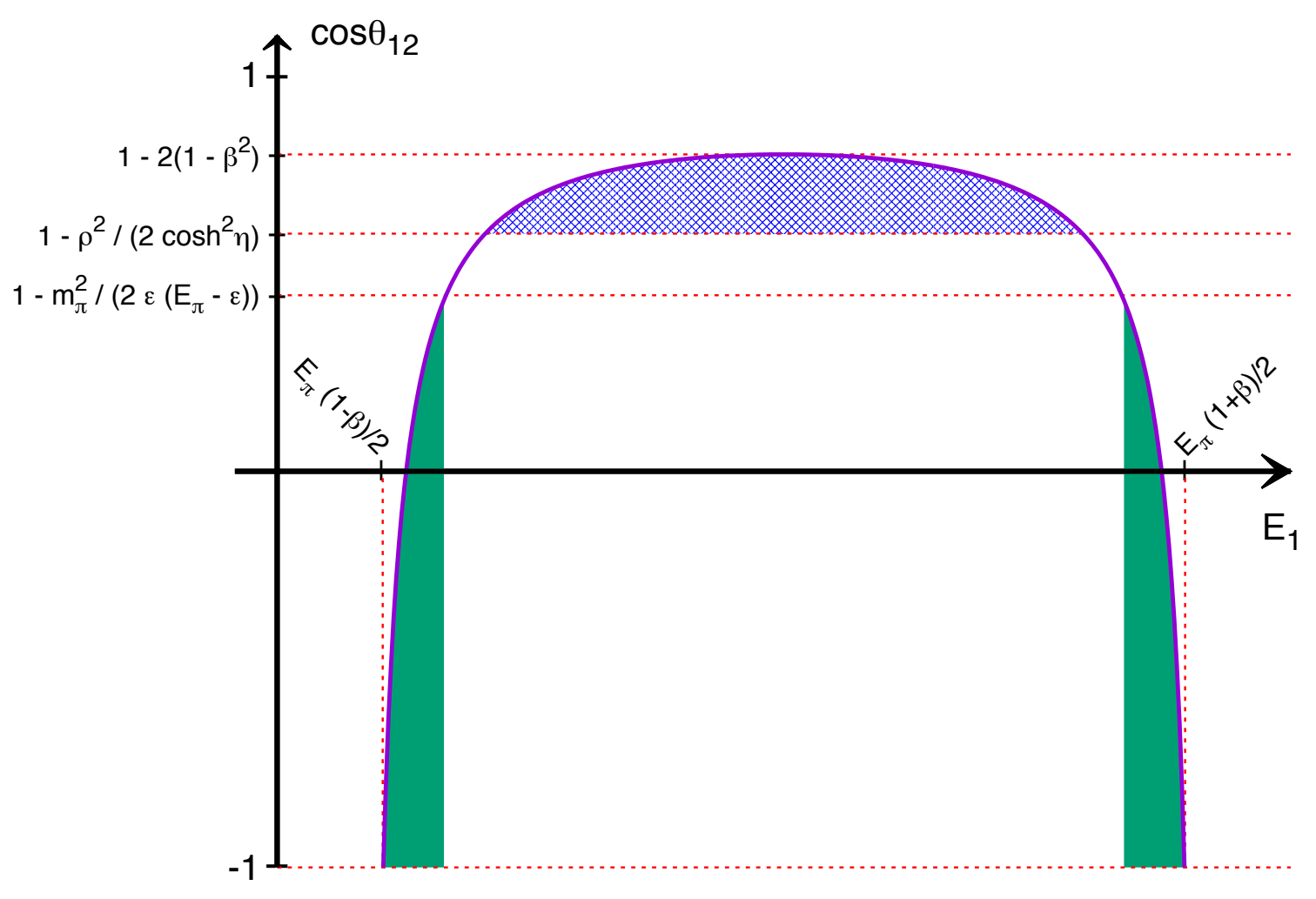

Figure 1: The cosine of the angle between the two photons, $\cos \theta_{12}$, as a function of the photon energy $E_{1}$, according to Eq. (16). The shaded areas correspond to B2 while the hatched one describes B1.

To obtain a better understanding of the phase space for the two backgrounds and how they are connected, it is instructive to plot $\cos \theta_{12}$ as a function of $E_{1}$ according to Eq. (16). This is done in Fig. 1. Without any restrictions, the region underneath the solid line would be the available phase space for the two photon decay. If the pion could be reconstructed experimentally in this whole region, there would be no pion decay background. As described above, the experimental limitations lead to some regions where only one electromagnetic signal is seen. In the upper hatched region representing B1 the two photons are close together and cannot be seen as separate photons by the detector. In the shaded regions (B2) left and right, they are well separated in angle, but one of them has energy below threshold and is not seen either. The full background comes from the sum of these regions. Only in the the remaining part can both photons be seen and the parent pion be reconstructed. We note that Fig. 1 shows an idealized picture where we 
have chosen the pion energy and the parameters $\rho$ and $\varepsilon$ such that the main qualitative effects are well visible. If we were to use more realistic values, the plot would look close to a rectangular function, with $\cos \theta_{12}$ very closely reaching unity at the upper end. The hatched and shaded areas would shrink in size, although they still make numerically very sizable contributions as we shall see in the next section. We also note that, depending on the kinematics, the areas of B1 and B2 may overlap. Hence, in the numerical calculation one has to carefully avoid double counting.

We finally emphasize that our calculations of the pion backgrounds are not limited to photonin-jet production, but can also be applied to any other photon observable. Of course, one has to combine the decay results with the appropriate pion cross sections corresponding to the photon observable one is interested in. For example, to obtain a background estimation for single-inclusive photon production one would use a single-inclusive pion cross section and apply the expressions for B1 and B2.

\section{Phenomenological results}

It is most interesting to consider the cross section differential in $z_{\gamma}$ as it directly probes the $z$ dependence of the $D_{c}^{\gamma}$. The upper part of Fig. 2 (solid line) shows the cross section for $p p \rightarrow$ (jet $\gamma) X$ at $\sqrt{S}=200 \mathrm{GeV}$, corresponding to RHIC. In the lower part we show results for $p p$ collisions at the LHC at $\sqrt{S}=7 \mathrm{TeV}$. We use the CT10 [31] parton distributions and the "GlückReya-Vogt" (GRV) photon fragmentation functions of Ref. [8]. The results have been integrated over $\left|\eta^{\text {jet }}\right|<1$ and over $5 \mathrm{GeV}<p_{T}^{\text {jet }}<30 \mathrm{GeV}$ for RHIC and $15 \mathrm{GeV}<p_{T}^{\text {jet }}<30 \mathrm{GeV}$ for the LHC. We also impose a cut $p_{T}>5 \mathrm{GeV}$ on the photon transverse momentum, in order to make sure that we are in the perturbative regime. As it turns out, this cut also helps to suppress the pion decay background (for the LHC the cut causes the "edges" in the figure at $z_{\gamma}=1 / 3$ where $5 \mathrm{GeV} / z_{\gamma}$ coincides with the lower limit of the $p_{T}^{\text {jet }}$ integration). The jet is always defined by the anti- $k_{t}$ algorithm with $R=0.6$. We choose $\mu_{R}=\mu_{F}=p_{T}^{\text {jet }}$ for the renormalization and the initial state factorization scales. The final state factorization scale, on the other hand, is set to

$\mu_{F}^{\prime \prime}=p_{T}^{\text {jet }} \sqrt{1-z_{\gamma}}$ (see also $\left.[16,17]\right)$. Along with our value of $R$ this choice helps to minimize the logarithm in Eq. (13) and hence the direct contribution to the cross section.

In addition to the cross sections we also show the estimated $\pi^{0}$ background in Fig. 2, separately for B1 and B2. Here we use the most recent set of pion fragmentation functions of [32]. We choose the detector resolutions $\delta \phi=\delta \eta \equiv \delta=0.01$ in (18), which are typical values for the RHIC and LHC collider experiments. We furthermore assume two different values for the energy threshold for photon detection, $\varepsilon=100 \mathrm{MeV}$ and $\varepsilon=10 \mathrm{MeV}$. We observe that for our choice of kinematics background $\mathrm{B} 1$ is small for $\delta \phi=\delta \eta=0.01$, since the two photons start to be seen as a single one only when their combined transverse momentum exceeds $\sim 20 \mathrm{GeV}$. One furthermore sees that background B2 remains below the photon cross section only for the relatively low threshold $\varepsilon=10 \mathrm{MeV}$. This evidently poses a challenge to experimental studies of photon-in-jet production.

Figure 3 analyzes the relative sizes of the contributions of quark/antiquark (summed over all flavors) and gluon fragmentation to the cross section, using the RHIC kinematical setup of Fig. 2. We have normalized all contributions to the LO cross section with GRV fragmentation 
$|\eta|<1, \mathrm{p}_{\mathrm{T}}>5 \mathrm{GeV}$, anti $\mathrm{k}_{\mathrm{t}}, \mathrm{R}=0.6, \mathrm{CT} 10, \mathrm{GRV} / \mathrm{DSS} 14$

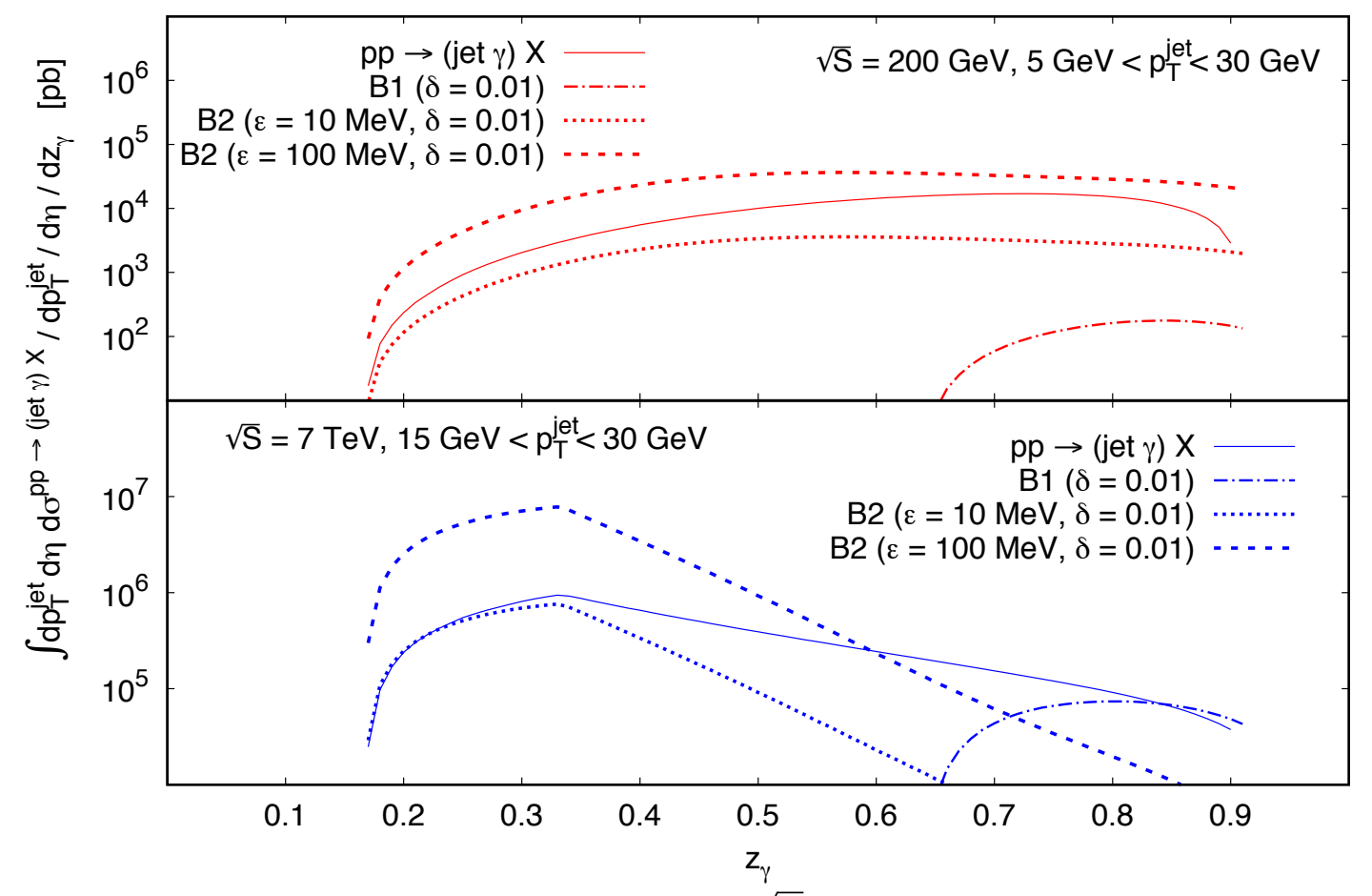

Figure 2: NLO cross section for $p p \rightarrow($ jet $\gamma) X$ at $\sqrt{S}=200 \mathrm{GeV}$ as function of $z_{\gamma}$, for $5 \mathrm{GeV}$ $<p_{T}^{\text {jet }}<30 \mathrm{GeV}$ (upper, solid), and at $\sqrt{S}=7 \mathrm{TeV}$ for $15 \mathrm{GeV}<p_{T}^{\text {jet }}<30 \mathrm{GeV}$ (lower, solid). The other lines show in both cases the backgrounds B1 and B2 from $\pi^{0}$ decay, for various choices of the detection parameters.

functions. We show NLO results for three different sets of photon fragmentation functions: the GRV [8] one used so far, and the two sets of "Bourhis, Fontannaz, Guillet", Ref. [9], which we refer to as "BFG1" and "BFG2" and properly transform to the DIS ${ }_{\gamma}$ scheme. One can see that overall gluon fragmentation makes a sizable contribution to the photon cross section. The BFG2 set is characterized by an especially large gluon-to-photon fragmentation function, which becomes clearly visible in the figure. We also show the direct contribution based on Eq. (13) which, in the DIS $_{\gamma}$ scheme and for our choice of $R$ and $\mu_{F}^{\prime \prime}$, is tiny. The cross section for photons produced in jets thus offers a direct probe of photon fragmentation also at NLO.

\section{Conclusions}

We have proposed a method of accessing the elusive photon fragmentation functions in hadronic collisions at RHIC or the LHC. The idea is to identify photons produced inside fully reconstructed jets, with the photon treated as part of the jet. The variable $z_{\gamma}=p_{T} / p_{T}^{\text {jet }}$ introduced in Eq. (2) allows to map out the $z$-dependence of the photon fragmentation functions. We have presented numerical results for the corresponding cross section that demonstrate the sensitivity to the fragmentation functions. We have also performed detailed estimates of the background 


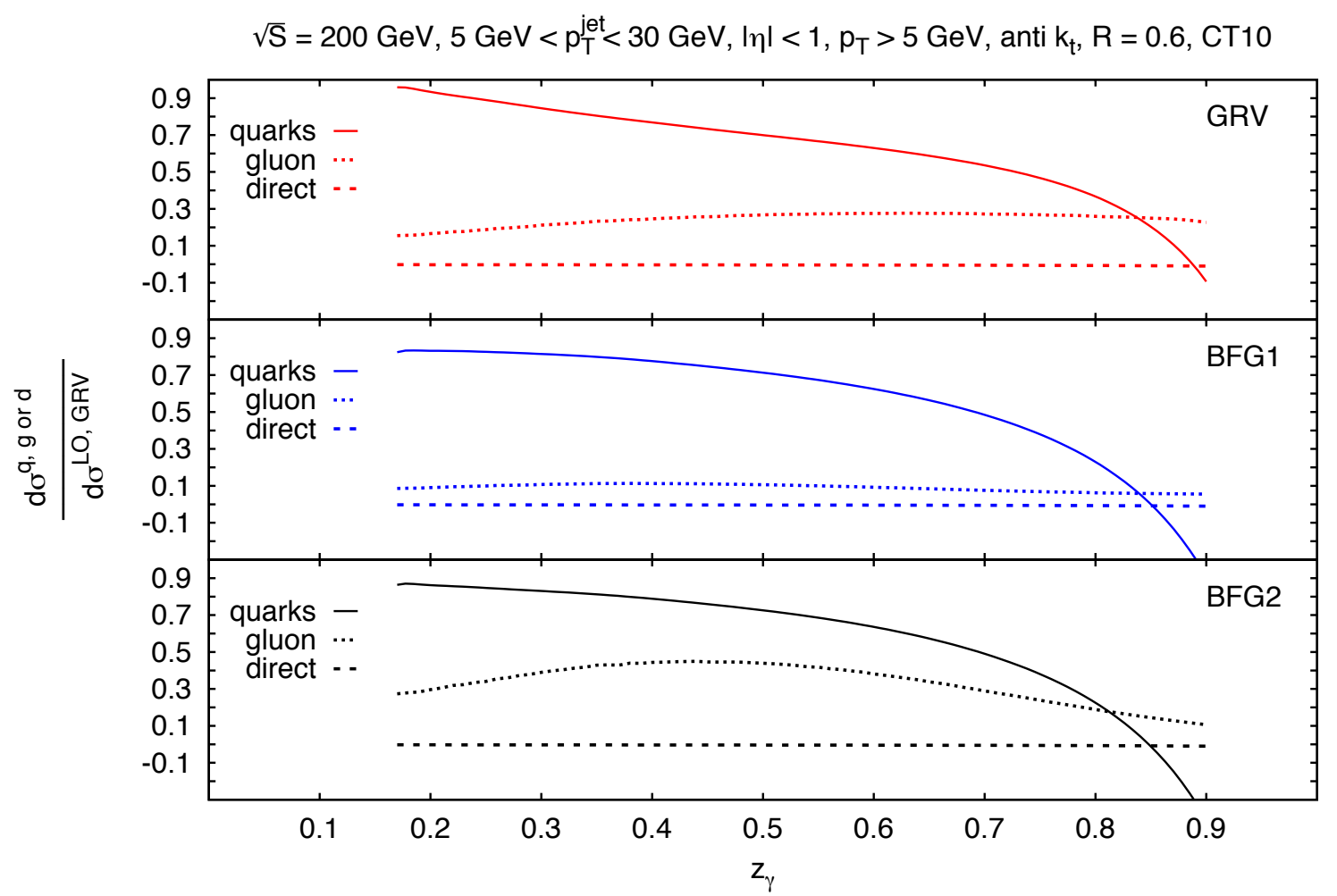

Figure 3: Normalized quark (solid) and gluon (dotted) fragmentation contributions together with the direct contribution (dashed) to the cross sections as functions of $z_{\gamma}$ for $5 \mathrm{GeV}<p_{T}^{\text {jet }}<30 \mathrm{GeV}$ at $\sqrt{S}=200 \mathrm{GeV}$. We show results for the sets of fragmentation functions of Refs. [8, 9]. For purpose of clearer presentation, all results have been normalized to the LO cross section obtained for GRV fragmentation functions.

from neutral-pion decay. These suggest that the measurement should be feasible provided a low photon detection threshold can be chosen. This will evidently present a significant challenge; on the other hand, there are likely further techniques available in experiment that allow to suppress the background, such as subtraction of the background using a measured pion-in-jet cross section. We are thus optimistic that clean measurements of the $D_{c}^{\gamma}$ should become possible in $p p$ collisions, enabling better theoretical control of collider observables involving high energy photons. We finally note that detailed future studies of the interplay of QCD radiation inside jets with photon production may also lead to new techniques for photon isolation.

\section{Acknowledgments}

We acknowledge most useful discussions with C. Gagliardi and Y. Akiba. This work was supported in part by the Bundesministerium für Bildung und Forschung (BMBF) grant 05P15VTCA1. 


\section{A Jet Functions}

For convenience we collect all NLO jet functions in the $\overline{\mathrm{MS}}$ scheme:

Jet functions $j$ :

$$
\begin{aligned}
j_{\gamma \rightarrow \gamma}(z, \lambda) & =\delta(1-z)+\mathcal{O}(\alpha) \\
j_{\gamma \rightarrow q}(z, \lambda) & =-\frac{\alpha e_{q}^{2}}{2 \pi}\left[P_{q \gamma}(z) \log \left(\lambda^{2}(1-z)^{2}\right)+z(1-z)\right], \\
j_{q \rightarrow \gamma}(z, \lambda) & =-\frac{\alpha e_{q}^{2}}{2 \pi}\left[P_{\gamma q}(z) \log \left(\lambda^{2}(1-z)^{2}\right)+z\right], \\
j_{\gamma \rightarrow g}(z, \lambda) & =\mathcal{O}\left(\alpha \alpha_{s}\right), \\
j_{g \rightarrow \gamma}(z, \lambda) & =\mathcal{O}\left(\alpha \alpha_{s}\right), \\
j_{(c \neq \gamma) \rightarrow(e \neq \gamma)}(z, \lambda) & =\delta(1-z) \delta_{e c}+\frac{\alpha_{s}}{2 \pi} f_{c \rightarrow e}(z, \lambda)+\mathcal{O}\left(\alpha_{s}^{2}\right)+\mathcal{O}\left(\alpha \alpha_{s}\right) .
\end{aligned}
$$

Jet functions $\tilde{j}$ :

$$
\begin{aligned}
\tilde{j}_{\gamma \rightarrow \gamma}(z, \lambda) & =\delta(1-z)+\mathcal{O}(\alpha), \\
\tilde{j}_{\gamma \rightarrow q}(z, \lambda) & =\frac{\alpha e_{q}^{2}}{2 \pi}\left[P_{q \gamma}(z) \log \left(\lambda^{2}(1-z)^{2}\right)+z(1-z)+\mathcal{I}_{q \gamma}^{\text {algo }}(z)\right], \\
\tilde{j}_{q \rightarrow \gamma}(z, \lambda) & =\frac{\alpha e_{q}^{2}}{2 \pi}\left[P_{\gamma q}(z) \log \left(\lambda^{2}(1-z)^{2}\right)+z+\mathcal{I}_{\gamma q}^{\text {algo }}(z)\right], \\
\tilde{j}_{\gamma \rightarrow g}(z, \lambda) & =\mathcal{O}\left(\alpha \alpha_{s}\right), \\
\tilde{j}_{g \rightarrow \gamma}(z, \lambda) & =\mathcal{O}\left(\alpha \alpha_{s}\right), \\
\tilde{j}_{(e \neq \gamma) \rightarrow\left(c^{\prime} \neq \gamma\right)}(z, \lambda) & =\delta(1-z) \delta_{e c^{\prime}}+\frac{\alpha_{s}}{2 \pi} \tilde{f}_{e \rightarrow c^{\prime}}(z, \lambda)+\mathcal{O}\left(\alpha_{s}^{2}\right)+\mathcal{O}\left(\alpha \alpha_{s}\right),
\end{aligned}
$$

with

$$
\mathcal{I}_{c^{\prime} c}^{\text {algo }}(z)=\left\{\begin{array}{cl}
2 P_{c^{\prime} c}(z) \log \left(\frac{z}{1-z}\right) \Theta(1 / 2-z) & \text { cone algorithm }, \\
2 P_{c^{\prime} c}(z) \log z & (\text { anti- }) k_{t} \text { algorithm }, \\
P_{c^{\prime} c}(z)\left[\log (z)+\log \left(\frac{z}{1-z}\right) \Theta(1 / 2-z)\right] & J_{E_{T}} \text { algorithm } .
\end{array}\right.
$$

The last lines in each of Eqs. (A.1) and (A.2) describe the "pure-QCD" type jet functions without any photonic contributions. They are shown only for the sake of completeness and listed explicitly in [14], from which the functions $f_{c \rightarrow e}(z, \lambda)$ and $\tilde{f}_{e \rightarrow c^{\prime}}(z, \lambda)$ may be read off easily.

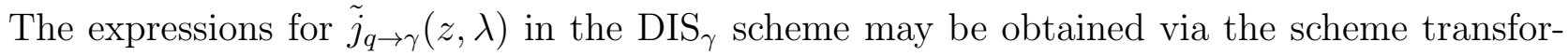
mation as shown in Eq. (13). All other jet functions are unaffected by that transformation. 


\section{References}

[1] CMS Collaboration, CMS-PAS-EXO-15-004; ATLAS collaboration, ATLAS-CONF-2015-081.

[2] J. F. Owens, Rev. Mod. Phys. 59, 465 (1987).

[3] P. Aurenche, A. Douiri, R. Baier, M. Fontannaz and D. Schiff, Phys. Lett. B 140, 87 (1984); P. Aurenche, R. Baier, M. Fontannaz and D. Schiff, Nucl. Phys. B 297, 661 (1988); H. Baer, J. Ohnemus and J. F. Owens, Phys. Rev. D 42, 61 (1990); L. E. Gordon and W. Vogelsang, Phys. Rev. D 48, 3136 (1993); L. E. Gordon and W. Vogelsang, Phys. Rev. D 50, 1901 (1994).

[4] F. Aversa, P. Chiappetta, M. Greco and J. P. Guillet, Nucl. Phys. B 327, 105 (1989); D. de Florian, Phys. Rev. D 67, 054004 (2003) [hep-ph/0210442]; B. Jäger, A. Schäfer, M. Stratmann and W. Vogelsang, Phys. Rev. D 67, 054005 (2003) [hep-ph/0211007].

[5] E. Laenen, G. Oderda and G. F. Sterman, Phys. Lett. B 438, 173 (1998) [hep-ph/9806467]; S. Catani, M. L. Mangano and P. Nason, JHEP 9807, 024 (1998) [hep-ph/9806484]; S. Catani, M. L. Mangano, P. Nason, C. Oleari and W. Vogelsang, JHEP 9903, 025 (1999) [hep-ph/9903436]; N. Kidonakis and J. F. Owens, Phys. Rev. D 61, 094004 (2000) [hepph/9912388]; G. F. Sterman and W. Vogelsang, JHEP 0102, 016 (2001) [hep-ph/0011289]; E. Laenen, G. F. Sterman and W. Vogelsang, Phys. Rev. Lett. 84, 4296 (2000) [hepph/0002078]; P. Bolzoni, S. Forte and G. Ridolfi, Nucl. Phys. B 731, 85 (2005) [hepph/0504115]; D. de Florian and W. Vogelsang, Phys. Rev. D 72, 014014 (2005) [hepph/0506150]; T. Becher and M. D. Schwartz, JHEP 1002, 040 (2010) [arXiv:0911.0681 [hep$\mathrm{ph}]$.

[6] D. Buskulic et al. [ALEPH Collaboration], Z. Phys. C 69, 365 (1996); K. Ackerstaff et al. [OPAL Collaboration], Eur. Phys. J. C 2, 39 (1998) [hep-ex/9708020].

[7] P. Aurenche, P. Chiappetta, M. Fontannaz, J. P. Guillet and E. Pilon, Nucl. Phys. B 399, 34 (1993).

[8] M. Glück, E. Reya and A. Vogt, Phys. Rev. D 48, 116 (1993) [Phys. Rev. D 51, 1427 (1995)].

[9] L. Bourhis, M. Fontannaz and J. P. Guillet, Eur. Phys. J. C 2, 529 (1998) [hep-ph/9704447].

[10] A. Gehrmann-De Ridder, T. Gehrmann and E. W. N. Glover, Phys. Lett. B 414, 354 (1997) [hep-ph/9705305]; A. Gehrmann-De Ridder and E. W. N. Glover, Nucl. Phys. B 517, 269 (1998) [hep-ph/9707224].

[11] W. Vogelsang and A. Vogt, Nucl. Phys. B 453, 334 (1995) [hep-ph/9505404].

[12] M. Glück, L. E. Gordon, E. Reya and W. Vogelsang, Phys. Rev. Lett. 73, 388 (1994).

[13] M. Klasen and F. Knig, Eur. Phys. J. C 74, no. 8, 3009 (2014) [arXiv:1403.2290 [hep-ph]].

[14] T. Kaufmann, A. Mukherjee and W. Vogelsang, Phys. Rev. D 92, 054015 (2015) [arXiv:1506.01415 [hep-ph]]. 
[15] F. Arleo, M. Fontannaz, J. P. Guillet and C. L. Nguyen, JHEP 1404, 147 (2014) [arXiv:1311.7356 [hep-ph]].

[16] Y. T. Chien, Z. B. Kang, F. Ringer, I. Vitev and H. Xing, arXiv:1512.06851 [hep-ph].

[17] M. Procura and W. J. Waalewijn, Phys. Rev. D 85, 114041 (2012) [arXiv:1111.6605 [hep-ph]].

[18] Z. Belghobsi, M. Fontannaz, J.-P. Guillet, G. Heinrich, E. Pilon and M. Werlen, Phys. Rev. D 79, 114024 (2009) [arXiv:0903.4834 [hep-ph]].

[19] E. W. N. Glover and A. G. Morgan, Z. Phys. C 62, 311 (1994).

[20] A. Gehrmann-De Ridder, G. Kramer and H. Spiesberger, Eur. Phys. J. C 11, 137 (1999) [hep-ph/9907511]; Nucl. Phys. B 578, 326 (2000) [hep-ph/0003082]; A. Gehrmann-De Ridder, T. Gehrmann and E. Poulsen, Eur. Phys. J. C 47, 395 (2006) [hep-ph/0604030].

[21] G. P. Salam and G. Soyez, JHEP 0705, 086 (2007) [arXiv:0704.0292 [hep-ph]].

[22] S. D. Ellis and D. E. Soper, Phys. Rev. D 48, 3160 (1993) [hep-ph/9305266]; S. Catani, Y. L. Dokshitzer, M. H. Seymour and B. R. Webber, Nucl. Phys. B 406, 187 (1993); M. Cacciari, G. P. Salam and G. Soyez, JHEP 0804, 063 (2008) [arXiv:0802.1189 [hep-ph]].

[23] H. Georgi, arXiv:1408.1161 [hep-ph]; Y. Bai, Z. Han and R. Lu, JHEP 1503, 102 (2015) [arXiv:1411.3705 [hep-ph]]; J. Thaler, Phys. Rev. D 92, no. 7, 074001 (2015) [arXiv:1506.07876 [hep-ph]].

[24] B. Jäger, M. Stratmann and W. Vogelsang, Phys. Rev. D 70, 034010 (2004) [hep-ph/0404057]; A. Mukherjee and W. Vogelsang, Phys. Rev. D 86, 094009 (2012) [arXiv:1209.1785 [hepph]]; T. Kaufmann, A. Mukherjee and W. Vogelsang, Phys. Rev. D 91, 034001 (2015) [arXiv:1412.0298 [hep-ph]].

[25] M. Dasgupta, F. A. Dreyer, G. P. Salam and G. Soyez, arXiv:1602.01110 [hep-ph].

[26] M. Glück, E. Reya and A. Vogt, Phys. Rev. D 45, 3986 (1992).

[27] K. A. Olive et al. [Particle Data Group Collaboration], Chin. Phys. C 38, 090001 (2014).

[28] A. Adare et al. [PHENIX Collaboration], Phys. Rev. D 86, 072008 (2012) [arXiv:1205.5533 [hep-ex]]; Y. Akiba, private communications.

[29] C. Gagliardi, private communications.

[30] J. Wess and B. Zumino, Phys. Lett. B 37, 95 (1971).

[31] H. L. Lai, M. Guzzi, J. Huston, Z. Li, P. M. Nadolsky, J. Pumplin and C.-P. Yuan, Phys. Rev. D 82, 074024 (2010) [arXiv:1007.2241 [hep-ph]].

[32] D. de Florian, R. Sassot, M. Epele, R. J. Hernández-Pinto and M. Stratmann, Phys. Rev. D 91, 014035 (2015) [arXiv:1410.6027 [hep-ph]]. 